\title{
Trends in mortality from idiopathic pulmonary fibrosis in the European Union: an observational study of the WHO mortality database from 2001-2013
}

\author{
Dominic C. Marshall (1) ${ }^{1}$, Justin D. Salciccioli ${ }^{2}$, Barry S. Shea ${ }^{3,5}$ and \\ Praveen Akuthota ${ }^{4,5}$
}

Affiliations: ${ }^{1}$ Oxford University Clinical Academic Graduate School, John Radcliffe Hospital, Oxford, UK. ${ }^{2}$ Dept of Medicine, Mount Auburn Hospital, Harvard Medical School, Cambridge, MA, USA. ${ }^{3}$ Division of Pulmonary, Critical Care and Sleep Medicine, Warren Alpert Medical School, Brown University, Providence, RI, USA. ${ }^{4}$ Division of Pulmonary, Critical Care, and Sleep Medicine, University of California San Diego, La Jolla, CA, USA. ${ }^{5}$ These authors contributed equally to the manuscript.

Correspondence: D.C. Marshall, Oxford University Clinical Academic Graduate School, Level 3, John Radcliffe Hospital, Oxford, OX3 9DU, UK. E-mail: dominic.marshallamedsci.ox.ac.uk

@ERSpublications

Reported IPF mortality is increasing across the EU with substantial variations between EU countries http://ow.ly/tpIO30h3hZK

Cite this article as: Marshall DC, Salciccioli JD, Shea BS, et al. Trends in mortality from idiopathic pulmonary fibrosis in the European Union: an observational study of the WHO mortality database from 2001-2013. Eur Respir J 2018; 51: 1701603 [https://doi.org/10.1183/13993003.01603-2017].

ABSTRACT Idiopathic pulmonary fibrosis (IPF) is the most common of the idiopathic interstitial pneumonias and is characterised by progressive accumulation of scar tissue in the lungs. The objective of this study was to describe the current mortality rates due to IPF in Europe, based on the World Health Organization (WHO) mortality database.

We used country-level data for IPF mortality, identified in the WHO mortality database using International Classification of Diseases 10th Edition (ICD-10) codes, for the period 2001-2013. Joinpoint analysis was performed to describe trends throughout the observation period.

The median mortality was 3.75 per 100000 (interquartile range (IQR) $1.37-5.30$ ) and 1.50 per 100000 (IQR 0.65-2.02) for males and females, respectively. IPF mortality increased in the majority of the European Union (EU) countries with the exceptions of Denmark, Croatia, Austria and Romania. There was a significant disparity in rates across Europe, in the range 0.41-12.1 per 100000 for men and $0.24-$ 5.63 per 100000 for women. The most notable increases were observed in the United Kingdom and Finland. Rates were also substantially higher in males, with sex disparity increasing across the period.

The reported IPF mortality appears to be increasing across the EU; however, there is substantial variation in mortality trends and overall reported mortality rates between countries.

Received: Aug 062017 | Accepted after revision: Oct 152017

Conflict of interest: Disclosures can be found alongside this article at erj.ersjournals.com

Copyright OERS 2018 


\section{Introduction}

Idiopathic pulmonary fibrosis (IPF) is a chronic degenerative lung disease and the most common of the idiopathic interstitial pneumonias [1,2]. It is diagnosed and distinguished from other interstitial lung diseases (ILDs) by a combination of clinical, radiological and histological findings. While smoking increases the risk of IPF [3], a clear aetiological origin is not known. Careful exclusion of environmental exposures (e.g. asbestos) or systemic diseases (e.g. rheumatoid arthritis) known to cause pulmonary fibrosis is an essential part of diagnosis. Though the novel antifibrotic agents pirfenidone [4] and nintedanib [5] have been recently developed to slow the progression of IPF, they do not cure the disease and outcomes remain poor.

The epidemiology of IPF shows the condition to be more common in males and with advancing age [6]. Previous studies have indicated that incidence and mortality rates from IPF in Europe and across the globe are increasing $[7,8]$. A systematic review assessing IPF epidemiology identified an annual incidence of 3-9 per 100000 for Europe [9]. Previous reports have demonstrated substantial geographical variation in IPF incidence, ranging from 1.3 per 100000 in Denmark [10] to 4.6 per 100000 in the United Kingdom [11]. Globally, there is thought to be significant variation in rates of diagnosis and reporting, potentially in part because of the availability of expertise and diagnostic equipment [12]. The diagnostic criteria for IPF have changed over time and were most recently updated in the 2011 consensus statement from the American Thoracic Society (ATS), European Respiratory Society (ERS), Japanese Respiratory Society (JRS) and Latin American Thoracic Association (ALAT) [13].

To the best of our knowledge, no up-to-date analysis has been published on IPF mortality across European Union (EU) member states. We have previously utilised the World health Organization (WHO) mortality database to analyse trends in mortality from ischaemic heart disease and cerebrovascular disease in Europe [14], and have applied similar methods to cancer mortality trends in the United Kingdom [15]. The objective of this study was to describe current mortality rates due to IPF in Europe, based on the WHO mortality database. Furthermore, we aimed to analyse trends in IPF mortality between 2001 and 2013 using Joinpoint regression analysis.

\section{Methods}

Data source

Mortality data from EU countries is extracted from the WHO mortality database for the years 2001-2013. Data quality is evaluated by the WHO which ensures reliability and provides details of reporting and comparability. For inclusion in the WHO mortality database, birth and population recording must exceed $90 \%$. The estimated level of coverage for death with a registered cause of death exceeds $97 \%$ in all EU countries included in the final analysis [16]. The estimated level of coverage is calculated by dividing the actual reporting rate by the estimated mortality rate. The estimated level of completeness for death with a registered cause of death was $100 \%$ in the majority of EU countries, with the exception of Croatia (98\%), the Czech Republic (99\%), Hungary (99\%), Romania (99\%) and Slovenia (99\%) [17, 18]. The estimated level of completeness is calculated by the WHO using demographic techniques such as the Brass Growth Balance Method [18].

Status as a European member state reporting mortality statistics using the International Classification of Diseases (ICD) 10th Edition (ICD-10) was the only inclusion criterion. Exclusion criteria were fewer than 1 million citizens and $>30 \%$ missing data for the observation period. We defined IPF using the ICD-10 code J84.1 as has previously been used in analysis of large mortality databases [9].

\section{Data handling}

Crude mortality rates per 100000 population were age standardised using the European Standard Population 2013. Sex-specific averages for the start and end of the observation period were calculated as the 3-year average age-standardised death rate (ASDR) for 2001-2003 and 2011-2013. Where there were missing data, 2-year averages were calculated. The percentage change over the observation period was calculated as the difference between the start and end averages.

\section{Statistical analysis}

Statistical trends were assessed using Joinpoint Version 4.1.1.1 (United States National Cancer Institute Surveillance Research Program). For the purpose of Joinpoint analysis, imputed data was used in a last observation carried forward manner. Joinpoint regression analysis assesses changes in linear slope for mortality trends over time, as described previously [14, 19]. Briefly, it assesses the overall trends in mortality, initially with no Joinpoints, and tests for significant changes in the model with sequential addition of points where there is significant change in the slope of the line. The model also computes an estimated annual percentage change (EAPC) for each trend by fitting a regression line to the natural 
logarithm of the rates. The log-linear transformation allows us to approximate normal distributions and, by estimating the annual percentage change, we are able to assess change in mortality trend at a constant percentage per year.

\section{Sensitivity analysis}

The diagnostic criteria for IPF have undergone recent revision and the potential overlapping nature of various interstitial pneumonias and other inflammatory fibrotic lung diseases have led previous reports to attempt the use of "narrow" and "broad" criteria in epidemiological studies of IPF [20]. For this reason, we chose to perform a single post hoc exploratory analysis to assess the influence of ICD coding for fibrosis from causes other than IPF. For this, we assessed mortality from the entire ICD-10 code J84 (other interstitial pneumonias) and each of its subcategories. We constructed figures from Joinpoint analysis to compare the contribution of IPF with the overall J84 classification.

\section{Results}

17 EU countries had sufficient data for analysis. Nine were excluded because of missing data and two had populations of less than one million citizens. Data are reported as mortality rate per 100000 standardised to the 2013 European standard population. Austria and Portugal adopted ICD-10 in 2002. We analysed the data by sex given the observed differences in overall and country-level mortality rates between females and males. The majority of individual EU countries demonstrated an increase in IPF mortality, with the exception of Denmark and Romania for females, and Denmark, Croatia and Austria for males (figure 1). The 3-year average mortality at the start and end of the observation period in each country, together with the percentage change over this period, is illustrated in table 1.

Median mortality for the last recorded observation was 3.80 per 100000 (interquartile range (IQR) 1.375.30) and 1.50 per 100000 (IQR 0.64-2.02) for males and females, respectively. For comparison, start and end 3-year averages and the percentage change across the period were computed. There was substantial variation across EU countries, with the highest rates at the end of the observation period in the UK (12.01 per 100000), Finland (7.36 per 100000) and Sweden (6.46 per 100000) for males. For females, the highest rates were in the UK (5.63 per 100000), Finland (3.62 per 100000) and Spain (3.35 per 100000). The lowest rates of IPF mortality for males were in Croatia (0.39 per 100000), Romania (0.64 per 100000$)$ and Lithuania (0.85 per 100000$)$. For females, the lowest rates were in Lithuania (0.24 per 100000), Romania (0.25 per 100000$)$ and Croatia (0.49 per 100000). Rates for males were predominantly higher than for females, with the exception of Croatia. The greatest difference between the male and the female mortality rate was found in Lithuania, with a 3.5-fold higher end 3-year average rate in men.

The largest percentage increase was in Lithuania for males $(+251.8 \%)$ and Croatia for females $(+270.1 \%)$. The absolute value increase was relatively small: 0.61 per 100000 for males in Lithuania and 0.26 per 100 000 for females in Croatia. A larger absolute increase occurred in the UK: 3.85 per 100000 and 2.02 per 100000 for males and females respectively. The greatest percentage decrease in mortality over our observation period was seen in Denmark for both males $(-47.2 \%)$ and females $(-54.9 \%)$. Changes in ASDR per 100000 are represented visually in figure 2.

Most countries demonstrated one continuous trend across the observation period. EAPC is calculated for each of the trends. Countries with a single increasing trend for both sexes include the Czech Republic (EAPC $+10.7 \%$ and $+10.5 \%$ for males and females, respectively), Finland (EAPC $+4.8 \%$ and $+2.99 \%$ for males and females, respectively), Germany (EAPC +4.7 and $+3.9 \%$ for males and females, respectively), Hungary (EAPC $+4.1 \%$ and $+1.9 \%$ for males and females, respectively), Lithuania (EAPC $+13.5 \%$ and $+9.5 \%$ for males and females, respectively), The Netherlands (EAPC $+3.3 \%$ and $+1.3 \%$ for males and females, respectively), Poland (EAPC $+6.1 \%$ and $+4.2 \%$ for males and females, respectively), Spain (EAPC $+2.3 \%$ and $+0.9 \%$ for males and females, respectively), Sweden (EAPC $+2.6 \%$ for both sexes) and the UK (EAPC $+3.8 \%$ and $4.0 \%$ for males and females, respectively).

The post hoc analysis to assess the contributions of IPF ( J84.1, narrow) to the overall ICD classification for total ILD-coded mortality ( J84, broad) across observed countries indicated trends for the broader category which largely reflected those of the narrower (figure 1).

\section{Discussion}

In this observational study of the WHO mortality database we report age-standardised death rates (ASDR) from IPF of 3.8 per 100000 for males and 1.5 per 100000 for females across the EU. There are substantial differences in IPF mortality rates between member states although there is an overall increase in mortality from IPF in most EU countries. Four countries (Denmark, Austria (males only), Croatia (males only) and Romania (females only)) demonstrated a decrease in mortality from IPF during the observation period. The greatest increases in IPF mortality were observed in the UK, Finland and Portugal. The greatest 

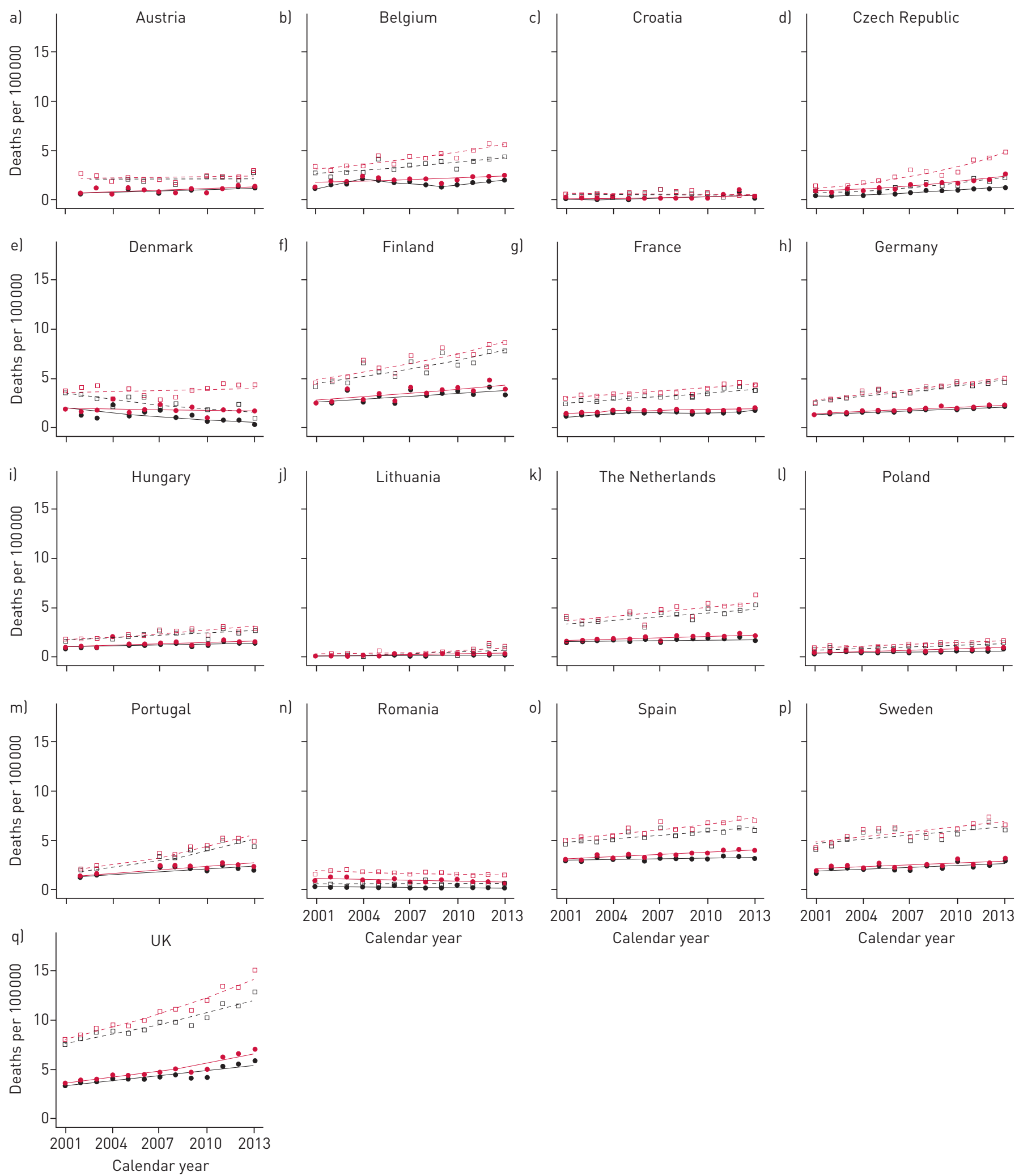

FIGURE 1 Mortality trends for males and females in 17 European countries. Lines represent the results of Joinpoint analyses while symbols

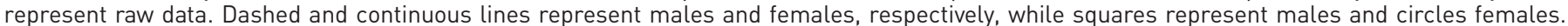
Black lines are for idiopathic pulmonary fibrosis (IPF; J84.1, narrow) and red lines for other interstitial pneumonias ( J84, broad). Where a symbol is absent data have been imputed for the respective year. 
TABLE 1 The first and last 3-year averages and percentage change in age standardised death rate (ASDR) for idiopathic pulmonary fibrosis (IPF) in 17 European countries during 2001-2013

\begin{tabular}{|c|c|c|c|c|c|c|c|c|}
\hline \multirow[t]{2}{*}{ Country } & \multicolumn{4}{|c|}{ Male } & \multicolumn{4}{|c|}{ Female } \\
\hline & 2001-2003 & 2011-2013 & Change ASDR & $\%$ Change & $2001-2003$ & 2011-2013 & Change ASDR & $\%$ Change \\
\hline Austria" & $2.56(8)$ & $2.34(7)$ & $-0.22(2)$ & $-8.5(3)$ & $0.9(6)$ & $1.29(7)$ & $0.39(8)$ & $+43.3(9)$ \\
\hline Belgium & $2.63(9)$ & $4.15(10)$ & $1.52(12)$ & $+57.6(11)$ & $1.43(12)$ & $1.88(11)$ & $0.45(11)$ & $+31.4(7)$ \\
\hline Croatia & $0.51(2)$ & $0.39(1)$ & $-0.13(3)$ & $-24.5(2)$ & $0.13(2)$ & $0.49(3)$ & $0.36(7)$ & $+270.1(17)$ \\
\hline Czech Republic & $0.77(5)$ & $2.13(6)$ & $1.36(11)$ & $+177.1(16)$ & $0.46(5)$ & $1.16(6)$ & $0.7(14)$ & $+150.4(16)$ \\
\hline Denmark & $3.28(12)$ & $1.73(5)$ & $-1.55(1)$ & $-47.2(1)$ & $1.39(10)$ & $0.63(4)$ & $-0.77(1)$ & $-54.8(1)$ \\
\hline Finland & $4.43(14)$ & $7.36(16)$ & $2.93(16)$ & $+66.3(13)$ & $2.92(15)$ & $3.62(16)$ & $0.70(15)$ & $+24.0(6)$ \\
\hline France & $2.63(10)$ & $3.97(9)$ & $1.34(10)$ & $+50.9(9)$ & $1.27(8)$ & $1.68(9)$ & $0.41(9)$ & $+32.1(8)$ \\
\hline Germany & $2.8(11)$ & $4.46(11)$ & $1.66(13)$ & $+59.4(12)$ & $1.43(11)$ & $2.08(12)$ & $0.66(13)$ & $+46.0(11)$ \\
\hline Hungary & $1.72(6)$ & $2.66(8)$ & $0.95(7)$ & $+55.1(10)$ & $0.97(7)$ & $1.39(8)$ & $0.42(10)$ & $+43.8(10)$ \\
\hline Lithuania & $0.24(1)$ & $0.85(3)$ & $0.61(6)$ & $+251.8(17)$ & $0.1(1)$ & $0.24(1)$ & $0.14(3)$ & $+148.1(15)$ \\
\hline The Netherlands & $3.56(13)$ & $4.81(13)$ & $1.24(8)$ & $+34.9(6)$ & $1.61(13)$ & $1.82(10)$ & $0.21(4)$ & $+12.8(4)$ \\
\hline Poland & $0.75(4)$ & $1.28(4)$ & $0.53(5)$ & $+71.5(14)$ & $0.44(4)$ & $0.68(5)$ & $0.24(5)$ & $+53.2(12)$ \\
\hline Portugal $\#$ & $2.11(7)$ & $4.77(12)$ & $2.67(15)$ & $+126.5(15)$ & $1.35(9)$ & $2.25(13)$ & $0.9(16)$ & $+66.6(14)$ \\
\hline Romania & $0.60(3)$ & $0.64(2)$ & $0.03(4)$ & $+5.5(4)$ & $0.34(3)$ & $0.25(2)$ & $-0.08(2)$ & $-25.2(2)$ \\
\hline Spain & $4.81(16)$ & $6.06(14)$ & $1.25(9)$ & $+26.0(5)$ & $3.02(16)$ & $3.35(15)$ & $0.32(6)$ & $+10.7(3)$ \\
\hline Sweden & $4.61(15)$ & $6.46(15)$ & $1.85(14)$ & $+40.1(7)$ & $2.11(14)$ & $2.59(14)$ & $0.48(12)$ & $+22.6(5)$ \\
\hline UK & $8.16(17)$ & $12.01(17)$ & $3.85(17)$ & $+47.2(8)$ & $3.61(17)$ & $5.63(17)$ & $2.02(17)$ & $+55.9(13)$ \\
\hline
\end{tabular}

Ranks for 2001-2003, 2011-2013, absolute change and percentage change are reported in parenthesis. \#: For Austria and Portugal data were missing for 2001, start of period was calculated as the average of 2002-2003 with percentage change being determined for 2002-2003 versus 2011-2013. 


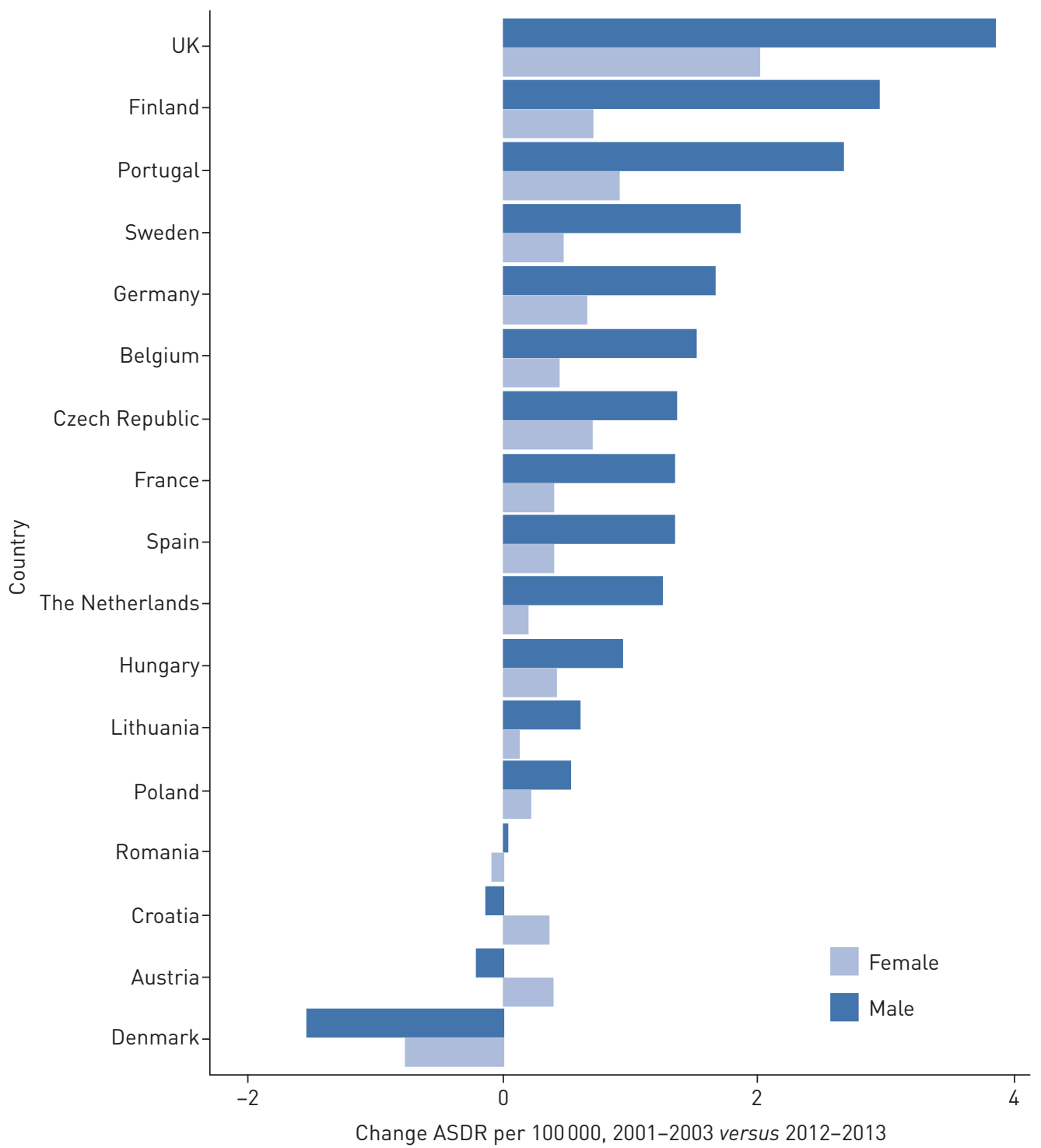

FIGURE 2 Ranked percentage change data comparing the average of European age-standardised death rate (ASDR) from idiopathic pulmonary fibrosis (IPF) for 2001-2003 versus 2011-2013 (unless otherwise specified). Data were missing for 2001 for Austria and Portugal and start of period was calculated as the average of 2002-2003, with percentage change being determined for 2002-2003 versus 2011-2013.

percentage increases occurred in countries that had comparatively low rates of IPF mortality, notably Lithuania, the Czech Republic and Croatia (females only). There is a persistent sex-mortality gap in most countries, a difference which appears to be increasing over time.

Our finding of increasing overall mortality attributed to IPF is consistent with previous reports $[8,21]$ and is likely to reflect an increase in the incidence of the disease across the EU. An increase in IPF incidence may be a true biological phenomenon, given the well-described ageing of the world population. Recent investigations have suggested that IPF is a disease of accelerated lung ageing [22] and cellular senescence, a hallmark of ageing, is increased in IPF lungs and appears to play an important role in disease pathogenesis [23]. Furthermore, aged mice are more susceptible to experimental lung fibrosis [24] and epidemiological data also indicate that the incidence of IPF rises with advancing age [6, 20,25].

Alternatively, the increasing mortality from IPF may be in keeping with increased recognition and diagnosis of the disease, as previously described [7, 26]. NAVARATNAm et al. [21] modelled IPF incidence using a UK General Practitioner database and identified an increase in the incidence of IPF. They also assessed death registry data and found consistent findings between the independent databases [21]. Levels of specialist knowledge and equipment for the diagnosis of IPF have increased with time and this may 
account for some of the observed increase in rates of diagnosis and reporting of mortality from this condition. Another potential contributing factor may be the spread of high-resolution scanners in the last two decades, which is reported to have contributed to increasing diagnosis rates [12]. Furthermore, Organisation for Economic Co-operation and Development (OECD) statistics indicate that the availability of computed tomography (CT) scanners has increased in Europe [27]. There have also been changes in diagnostic criteria for IPF over time, with the first guidelines published in 2000 and then modified in 2011 [13], which may further impact case identification.

An increase in mortality among the IPF population could also contribute to the increasing mortality rates observed; however, in the absence of a European-wide database, it is difficult to scientifically address this possibility. Although efforts have been made to generate a Europe-wide registry [28], the results are not yet comprehensive. While the WHO mortality database used in this study is unique in that it captures population-level mortality statistics, future investigations in mortality from IPF would be enhanced with a resource that provides similar population-level statistics on incidence and prevalence. Such a resource would allow investigators to assess whether changes in mortality statistics from IPF are driven primarily by increasing incidence of IPF or whether changes in mortality are limited to the IPF population alone. While the dataset used in the current study does not directly address IPF incidence, it may be a useful proxy for examining population-level diagnostic trends.

Previous studies have highlighted geographical variations in IPF mortality [8]. Potential explanations for the geographical variation may include environmental and genetic factors. Smoking is one of the most important risk factors for IPF; however, countries with higher IPF mortality rates have similar or lower rates of smoking to those with lower IPF mortality rates [29]. Despite the genetic similarity across EU member states, it is possible that genetic differences may contribute to the geographical variation in IPF incidence, as has been suggested elsewhere [30]. In addition, a study in the United States has demonstrated lower mortality from IPF in the black population [31]. KAUNISTO et al. have argued that regional differences are likely to be due to heterogeneity of data collection [32]; however, this heterogeneity is reported to be between studies rather than between countries using a unified database. Other approaches have involved using clinical registries and survey data, approaches which are likely to involve significant selection bias. These studies are, however, likely to have higher levels of case validation than is present in national death registries based on death certificate data.

In population studies IPF is frequently found to be more prevalent in males than in females [11, 20]. A previous report found similar mortality rates to our findings for the period 2005-2008 and supported a male-to-female mortality ratio of $\sim 2: 1$ in the UK [21]. In the current study, we observe a sex-mortality gap that is present in most EU countries and this sex-mortality difference appears to be increasing over time. Our finding of an increasing difference between men and women is in contrast to other health conditions, where we have observed a narrowing of sex-related mortality differences, for example in cardiovascular disease [14] and some cancers [15, 33]. However, whereas in most other health conditions a sex-mortality difference may be attributed to different patterns in smoking and hazardous drinking [34], previous studies have suggested that these behaviours do not contribute to IPF mortality [35].

There are several limitations that should be considered when interpreting the results of this study. As with any registry data, the accuracy of the mortality rates is dependent upon the quality of death certification and there is always potential for the true cause of death to be different from that recorded. Although we have chosen to use data from the WHO mortality database, which ensures data quality through careful evaluation prior to inclusion, coding practices may vary between countries and over time, potentially limiting the utility of geographic and temporal comparisons. Despite these potential limitations, the WHO database is a valuable resource for exploring diagnostic trends. While we acknowledge that there may be variation between countries in adopting new iterations of consensus diagnostic criteria, we have chosen to focus on changes over time in IPF mortality rates rather than differences between countries with respect to crude mortality rate. Furthermore, we restricted our analysis to ICD-10 codes and defined IPF as J84.1, which has been used previously for epidemiological studies of IPF [9]. Since these data reflect the study period 2001-2013, and given that the idiopathic interstitial pneumonias were reclassified in 2013 [36] and the diagnostic criteria were refined in 2011 [13], our report may reflect a heterogeneous group of ILDs rather than relating exclusively to IPF. An additional limitation of this study is that it is not likely to account for changes in IPF mortality due to the introduction of the novel antifibrotic agents pirfenidone and nintedanib. Pirfenidone was first approved for use in the EU in 2011 but was not available in most member states until 2012. Therefore, its effect on IPF mortality in the last observation period (2011-2013) is likely to be minimal. Furthermore, nintedanib was not approved in the EU until 2015 while, at the end of our observation period, the combination of prednisolone/azathioprine/ $\mathrm{N}$-acetylcysteine was demonstrated to increase mortality [37] and is no longer recommended. Any resulting decrease in mortality is thus unlikely to have been observed in these data. 
In summary, IPF mortality rates appear to be increasing across the EU and this increase may reflect a greater prevalence of the disease, driven in part by case identification. In the absence of a Europe-wide registry it is not possible to assess the survival of patients with IPF, thus highlighting the need to develop such a resource. IPF mortality rates are higher in males than in females and this disparity appears to be increasing in size. While this may reflect local coding practices, there is substantial variation between countries in the number of deaths recorded with IPF as the primary cause.

\section{Acknowledgements}

D.C. Marshall, J.D. Salciccioli and P. Akuthota were responsible for project planning and study design. D.C. Marshall and J.D. Salciccioli were responsible for data analysis. D.C. Marshall, J.D. Salciccioli, B.S. Shea and P. Akuthota were responsible for interpretation of results. All authors contributed to the first draft of the manuscript and have reviewed the final version before submission. The corresponding author affirms that the manuscript is an honest, accurate, and transparent account of the study being reported and that no important aspects of the study have been omitted.

\section{References}

1 Thomeer M, Demedts M, Vandeurzen K. Registration of interstitial lung diseases by 20 centres of respiratory medicine in Flanders. Acta Clin Bel 2001; 56: 163-172.

2 King TE, Pardo A, Selman M. Idiopathic pulmonary fibrosis. Lancet 2011; 378: 1949-1961.

3 Baumgartner KB, Samet JM, Stidley CA, et al. Cigarette smoking: a risk factor for idiopathic pulmonary fibrosis. Am J Respir Crit Care Med 1997; 155: 242-248.

4 King TE Jr, Bradford WZ, Castro-Bernardini S, et al. A phase 3 trial of pirfenidone in patients with idiopathic pulmonary fibrosis. N Engl J Med 2014; 370: 2083-2092.

5 Richeldi L, du Bois RM, Raghu G, et al. Efficacy and safety of nintedanib in idiopathic pulmonary fibrosis. $N$ Engl J Med 2014; 370: 2071-2082.

6 Ley B, Collard HR. Epidemiology of idiopathic pulmonary fibrosis. Clin Epidemiol 2013; 5: 483-492.

7 Sgalla G, Biffi A, Richeldi L. Idiopathic pulmonary fibrosis: diagnosis, epidemiology and natural history. Respirology 2016; 21: 427-437.

8 Hutchinson JP, McKeever TM, Fogarty AW, et al. Increasing global mortality from idiopathic pulmonary fibrosis in the twenty-first century. Ann Am Thorac Soc 2014; 11: 1176-1185.

9 Hutchinson J, Fogarty A, Hubbard R, et al. Global incidence and mortality of idiopathic pulmonary fibrosis: a systematic review. Eur Respir J 2015; 46: 795-806.

10 Hyldgaard C, Hilberg O, Muller A, et al. A cohort study of interstitial lung diseases in central Denmark. Respir Med 2014; 108: 793-799.

11 Gribbin J, Hubbard RB, Le Jeune I, et al. Incidence and mortality of idiopathic pulmonary fibrosis and sarcoidosis in the UK. Thorax 2006; 61: 980-985.

12 Caminati A, Madotto F, Cesana G, et al. Epidemiological studies in idiopathic pulmonary fibrosis: pitfalls in methodologies and data interpretation. Eur Respir Rev 2015; 24: 436-444.

13 Raghu G, Collard HR, Egan JJ, et al. An official ATS/ERS/JRS/ALAT statement: idiopathic pulmonary fibrosis: evidence-based guidelines for diagnosis and management. Am J Respir Crit Care Med 2011; 183: 788-824.

14 Hartley A, Marshall DC, Salciccioli JD, et al. Trends in mortality from ischaemic heart disease and cerebrovascular disease in Europe: 1980-2009. Circulation 2016; 133: 1916-1926.

15 Marshall DC, Webb TE, Hall RA, et al. Trends in UK regional cancer mortality 1991-2007. Br J Cancer 2016; 114: 340-347.

16 Census and civil registration coverage data by country. Geneva, World Health Organization, 2017. http://apps.who. int/gho/data/node.main.121?lang=enWHO Date last updated: April 24, 2017. Date last accessed: November 01, 2017.

17 WHO methods and data sources for country-level causes of death 2000-2012. Geneva, World Health Organization, 2014. www.who.int/healthinfo/statistics/GlobalCOD_method.pdf Date last accessed: December 06, 2017.

18 WHO methods and data sources for country-level causes of death 2000-2015. Geneva, World Health Organization, 2017. www.who.int/healthinfo/global_burden_disease/GlobalCOD_method_2000_2015.pdf Date last accessed: November 01, 2017.

19 Kim H, Fay MP, Feuer EJ, et al. Permutation tests for Joinpoint regression with applications to cancer rates. Stat Med 2000; 19: 335-351.

20 Raghu G, Weycker D, Edelsberg J, et al. Incidence and prevalence of idiopathic pulmonary fibrosis. Am J Respir Crit Care Med 2006; 174: 810-816.

21 Navaratnam V, Fleming KM, West J, et al. The rising incidence of idiopathic pulmonary fibrosis in the UK. Thorax 2011; 66: 462-467.

22 Selman M, Pardo A. Revealing the pathogenic and aging-related mechanisms of the enigmatic idiopathic pulmonary fibrosis. An integral model. Am J Respir Crit Care Med 2014; 189: 1161-1172.

23 Schafer MJ, White TA, Iijima K, et al. Cellular senescence mediates fibrotic pulmonary disease. Nat Commun 2017; 8: 14532 .

24 Redente EF, Jacobsen KM, Solomon JJ, et al. Age and sex dimorphisms contribute to the severity of bleomycin-induced lung injury and fibrosis. Am J Physiol Cell Mol Physiol 2011; 301: L510-L518.

25 Raghu G, Chen S-Y, Yeh W-S, et al. Idiopathic pulmonary fibrosis in US Medicare beneficiaries aged 65 years and older: incidence, prevalence, and survival, 2001-11. Lancet Respir Med 2014; 2: 566-572.

26 Olson AL, Swigris JJ. Idiopathic pulmonary fibrosis: diagnosis and epidemiology. Clin Chest Med 2012; 33: 41-50.

27 Computed tomography (CT) scanners. Paris, OECD, 2016. https://data.oecd.org/healtheqt/ computed-tomography-ct-scanners.htm Date last accessed: November 01, 2017.

28 European IPF Registry and Biobank. Giessen, European IPF Network, 2008. www.pulmonary-fibrosis.net. Date last accessed: November 01, 2017. 
$29 \mathrm{Ng} \mathrm{M}$, Freeman MK, Fleming TD, et al. Smoking prevalence and cigarette consumption in 187 countries, 1980-2012. JAMA 2014; 311: 183-192.

30 Natsuizaka M, Chiba H, Kuronuma K, et al. Epidemiologic survey of Japanese patients with idiopathic pulmonary fibrosis and investigation of ethnic differences. Am J Respir Crit Care Med. 2014; 190: 773-779.

31 Olson AL, Swigris JJ, Lezotte DC, et al. Mortality from pulmonary fibrosis increased in the United States from 1992 to 2003. Am J Respir Crit Care Med. 2007; 176: 277-284.

32 Kaunisto J, Salomaa E, Hodgson U, et al. Idiopathic pulmonary fibrosis-a systematic review on methodology for the collection of epidemiological data. BMC Pulm Med 2013; 13: 53.

33 Abotchie PN, Vernon SW, Du XL. Gender differences in colorectal cancer incidence in the United States, 1975 2006. J Womens Health (Larchmt) 2012; 21: 393-400.

34 McCartney G, Mahmood L, Leyland AH, et al. Contribution of smoking-related and alcohol-related deaths to the gender gap in mortality: evidence from 30 European countries. Tob Control 2011; 20: 166-168.

35 Ley B, Ryerson CJ, Vittinghoff E, et al. A multidimensional index and staging system for idiopathic pulmonary fibrosis. Ann Intern Med 2012; 156: 684-691.

36 Travis WD, Costabel U, Hansell DM, et al. An official American Thoracic Society/European Respiratory Society statement: update of the international multidisciplinary classification of the idiopathic interstitial pneumonias. Am J Respir Crit Care Med 2013; 188: 733-748.

37 Idiopathic Pulmonary Fibrosis Clinical Research Network, Raghu G, Anstrom KJ, et al. Prednisone, azathioprine, and N-acetylcysteine for pulmonary fibrosis. N Engl J Med. 2012; 366: 1968-1977. 